\title{
Molecular cytogenetic identification of three rust-resistant wheat-Thinopyrum ponticum partial amphiploids
}

\author{
Yanru Pei ${ }^{1}$, Yu Cui ${ }^{1}$, Yanping Zhang ${ }^{2}$, Honggang Wang ${ }^{1,2}$, Yinguang Bao ${ }^{2}$ and Xingfeng $\mathrm{Li}^{1,2^{*}}$
}

\begin{abstract}
Background: Thinopyrum ponticum $\left(2 n=10 x=70, s^{5} J^{5} s^{5} \rho^{5} J \jmath J J\right)$ is an important wild perennial Triticeae species that has a unique gene pool with many desirable traits for common wheat. The partial amphiploids derived from wheat-Th. ponticum set up a bridge for transferring valuable genes from Th. ponticum into common wheat.

Results: In this study, genomic in situ hybridization (GISH), multicolor GISH (mcGISH) and fluorescence in situ hybridization (FISH) were used to analyze the genomic constitution of SN0389, SN0398 and SN0406, three octoploid accessions with good resistance to rust. The results demonstrated that the three octoploids possessed 42 wheat chromosomes, while SN0389 contained 12 Th. ponticum chromosomes and SN0398 and SN0406 contained 14 Th. ponticum chromosomes. The genomic constitution of SN0389 was $42 \mathrm{~W}+12 J^{\mathrm{S}}$, and for SN0398 and SN0406 it was $42 \mathrm{~W}+$ $12 \mathrm{~J}^{\mathrm{S}}+2 \mathrm{~J}$. Chromosomal variation was found in chromosomes 1A, 3A, 6A, 2B, 5B, 6B, 7B, 1D and 5D of SN0389, SN0398 and SN0406 based on the FISH and McGISH pattern. A resistance evaluation showed that SN0389, SN0398 and SN0406 possessed good resistance to stripe and leaf rust at the seedling stage and adult-plant stage.
\end{abstract}

Conclusions: The results indicated that these wheat-Th. ponticum partial amphiploids are new resistant germplasms for wheat improvement.

Keywords: Thinopyrum ponticum, Common wheat, Partial amphiploids, In situ hybridization, Stripe rust, Leaf rust

\section{Background}

Thinopyrum ponticum (Podp.) Barkworth \& D.R. Dewey [syn. Agropyron elongatum (Host) P. Beauv., Lophopyrum ponticum (Popd.) A. Löve, Elytrigia pontica (Popd.) Holub] $(2 \mathrm{n}=10 \mathrm{x}=70)$, a perennial Triticeae species that is closely related to wheat, has been used for more than half a century to enrich the wheat germplasm with desirable traits [3]. Many important genes have been successfully transferred to common wheat from Th. ponticum, including resistance to powdery mildew [18], stripe rust [12, 30], leaf rust [24], stem rust [7, 23], Fusarium head blight $[9,14,26,27]$ and wheat streak mosaic virus [17], as well as abiotic stress tolerance [4, $28]$, and even yield-related traits [16, 21]. Although it is widely used in wheat improvement, the genomic

\footnotetext{
* Correspondence: lixf@sdau.edu.cn

${ }^{1}$ State Key Laboratory of Crop Biology, Shandong Agriculture University, Tai'an 271018, Shandong, China

${ }^{2}$ College of Agronomy, Shandong Agriculture University, Tai'an 271018, Shandong, China
}

composition of Th. ponticum has been long debated. Past research suggests that Th. ponticum is a decaploid with the genome formula JJJJJJJJJ [22]. Using St genomic DNA from the diploid Pseudoroegneria strigosa as a probe and the E genomic DNA from Th. elongatum for blocking, Chen et al. [3] revealed that the genomic composition of Th. ponticum was J $J^{\mathrm{S}} \mathrm{J}^{\mathrm{S}} \mathrm{S}^{\mathrm{S}} \mathrm{S}^{\mathrm{S}} \mathrm{TJJJJ}$. The J genome of Th. ponticum is homologous to the J genome of the diploid Thinopyrum bessarabicum, while the $\mathrm{J}^{\mathrm{S}}$ genome is a modified J genome of unknown origin [3].

Stripe rust and leaf rust are both severe foliar diseases in wheat (Triticum aestivum L.) all over the world. Stripe rust is caused by the fungus Puccinia striiformis $\mathrm{f}$. sp. Tritici., and it can cause severe yield loss in common wheat [25]. Leaf rust is caused by Puccinia recondita $f$. $s p$. Tritici., which is the most widespread and regularly occurring rust on wheat and can also cause yield losses up to $50 \%$ in extremely susceptible cultivars [6]. Breeding resistant cultivars is the most effective and economical means to control the disease [13]. At the present 
time, many of the existing resistance genes have been overcome by newly emerged virulent isolates. Thus, it is necessary and pressing to exploit new resistant genes for wheat breeding.

Genomic in situ hybridization (GISH) is widely used and is an effective means for detecting alien chromosomes and chromosome segments in wheat-alien species amphiploids, addition lines, and translocation lines. Multicolor GISH (mcGISH) is used to discriminate the $\mathrm{A}, \mathrm{B}, \mathrm{D}$ and $\mathrm{E}$ genomes of wheat - Th. ponticum addition, substitution and translocation lines [9, 10]. Fluorescence in situ hybridization (FISH), which uses repetitive DNA clones or oligonucleotides as a probe, is an extremely useful method for identifying chromosomes within a species or detecting intergenomic chromosome rearrangements in a polyploid species $[5,15,19]$.

In this study, three novel wheat-Th. ponticum partial amphiploids were developed from derivatives of common wheat and Th. ponticum, and FISH, GISH and mcGISH analyses were used to identify their genomic constitution. Furthermore, the resistance to stripe and leaf rust of the three partial amphiploids was also identified.

\section{Methods}

\section{Plant materials}

The plant materials used in this study included Th. ponticum, Pseudoroegneria spicata (StSt, 2n=14), Aegilops speltoides (SS, $2 \mathrm{n}=14)$, Aegilops tauschii $(\mathrm{DD}, 2 \mathrm{n}=14)$, the common wheat cultivar Yannong15 (YN15) and three wheat-Thinopyrum ponticum partial amphiploids (SN0389, SN0398 and SN0406). Among them, Th. ponticum was provided by Prof. Zhensheng Li (formerly of the Northwest Institute of Botany at the Chinese Academy of Sciences, Yangling, China). Ps. spicate, Triticum urartu, A. speltoides and A. tauschii were provided by Prof. Lihui Li from the Institute of Crop Science, Chinese Academy of Agricultural Sciences, Beijing, China. The partial amphiploids SN0389, SN0398 and SN0406 were selected from $\mathrm{BC}_{1} \mathrm{~F}_{7}$ of common wheat Yannong15 crossed with Th. ponticum, based on the stability and good phenotypic characteristics, such as long spikes, advanced fluorescence, and so on. The amphiploids were maintained by selfing in our laboratory.

\section{Mitotic and meiotic studies}

The seeds were germinated at $25{ }^{\circ} \mathrm{C}$ on moistened filter paper in petri dishes for $24 \mathrm{~h}$, were maintained at $4{ }^{\circ} \mathrm{C}$ for approximately 1 day, and were then transferred to $25{ }^{\circ} \mathrm{C}$ for approximately $12 \mathrm{~h}$. Roots, of a length of $1-2 \mathrm{~cm}$, were collected and immediately placed in ice water. After 24-32 h, these roots were fixed in Carnoy's solution for $24 \mathrm{~h}$ and were then stored in $70 \%(\mathrm{v} / \mathrm{v})$ ethanol. The root tips were squashed in acetic acid and were observed under a phase contrast microscope. When the flag leaf of the wheat was spread, the young spikes were sampled, and the anthers, at metaphase I (MI) of meiosis, were fixed in Carnoy's solution, dissociated in $1 \mathrm{M}$ $\mathrm{HCl}$ at $60{ }^{\circ} \mathrm{C}$ for $6-8 \mathrm{~min}$, and homogenized in $1 \%$ acetocarmine.

\section{Genomic in situ hybridization (GISH)}

Genomic DNA from Ps. spicata was labeled with Texas red-5-dCTP by the nick translation method and was used as a probe. Sheared genomic DNA from Yannong15 was used as the blocking DNA. The slides were counterstained with DAPI in Vectashield mounting medium (Vector Laboratories, USA). The detailed procedures of the chromosome spread preparation and hybridization are described by Bao et al. $[1,2]$. The $J^{\mathrm{S}}$ genomic and J-genomic chromosomes were distinguished by the GISH signals [3, 29], and those with centromeres labeled by the red signals were the $\mathrm{J}^{\mathrm{S}}$-genome and those with two arm ends of chromosomes labeled by signals were the J-genome.

\section{Multicolor genomic in situ hybridization (mcGISH)}

Total genomic DNA from T. urartu, A. speltoides and $A$. tauschii was isolated from the young leaves via a modified CTAB method. The total genomic DNA from $T$. urartu was labeled with fluorescein-12-dUTP, and the genomic DNA from A. tauschii was labeled with Texasred-5-dUCP by the nick translation method. Total genomic DNA from A. speltoides was used a blocker (at a ratio of 1:160). After hybridization, the slides were washed in $2 \times$ saline sodium citrate (SSC) and mounted in Vectashield mounting medium.

\section{Fluorescence in situ hybridization (FISH)}

Two probes were used in the multicolor FISH. pAs1 was labeled with fluorescein-12-dUTP, and the repeated DNA sequence, $(\mathrm{GAA})_{8}$, was labeled with Texas-red-5dUCP. Before hybridization, the two probes were mixed at a ratio of 4:1. The detailed procedures for the hybridization were previously described by $\mathrm{He}$ et al. [11]. Images were captured with an Olympus BX-60 fluorescence microscope equipped with a CCD (charge-coupled device) camera.

\section{Stripe rust and leaf rust resistance evaluation}

The stripe rust resistance of the three partial amphiploids, at the seedling stage, was evaluated with stripe rust race CYR32 in a greenhouse that had a favorable environment for stripe rust development at the Shandong Academy of Agricultural Sciences, Jinan, China. At the adult-plant stage, stripe rust and leaf rust resistance were evaluated under natural conditions. YN15 (the susceptible cultivar for stripe rust and leaf rust) and Th. ponticum were planted as contrasts at the 
same time. When the control variety YN15 was all fully infected, the evaluation results were scored according to the standard classification system with 6 scales from 0 to 4 as follows: 0 for no visible symptoms; 0 , for necrotic flecks without sporulation; and 1, 2, 3, and 4 for strongly resistant, resistant, susceptible and strongly susceptible, respectively.

\section{Results}

Chromosomal constitution of three partial amphiploids An analysis of the mitotic chromosomes showed that SN0389 contained a chromosome number of $2 n=54$, and both SN0398 and SN0406 had a mitotic chromosome number of $2 n=56$ (Fig. 1). The meiotic observations of the three partial amphiploids indicated that most of the chromosomes in the observed pollen mother cells of SN0389 formed 27 bivalents at meiotic MI, and SN0398 and SN0406 both formed into 28 bivalents, which proved that these three partial amphiploids exhibited high cytological stability.

GISH, mcGISH and FISH were used to analyze the genomic constitution of SN0389, SN0398 and SN0406. The results of the GISH (Fig. 1-A1) and FISH (Fig. 1-A3) analyses revealed that SN0389 had 42 wheat chromosomes and 12 Th. ponticum chromosomes, including six pairs of $\mathrm{J}^{\mathrm{S}}$-genome chromosomes (Fig. 2). SN0398 contained 42 wheat chromosomes and $14 \mathrm{Th}$. ponticum chromosomes (Fig. 1-B1, B2, B3), including six pairs of $\mathrm{J}^{\mathrm{S}}$-genome chromosomes and one pair of J-genome chromosomes (Fig. 2). The genomic constitution of SN0406 (Fig. 1-C1, C2, C3 and Fig. 2) was similar to SN0398. According to the configuration and signal of the alien chromosomes, the alien chromosomes of SN0389 were different compared to SN0398 and SN0406, while some of the alien chromosomes in SN0398 were probably identical to that in SN0406. For example, the $\mathrm{J}^{\mathrm{S}}-4^{*}, \mathrm{~J}^{\mathrm{S}}-6^{*}$ and $\mathrm{J}-1^{*}$
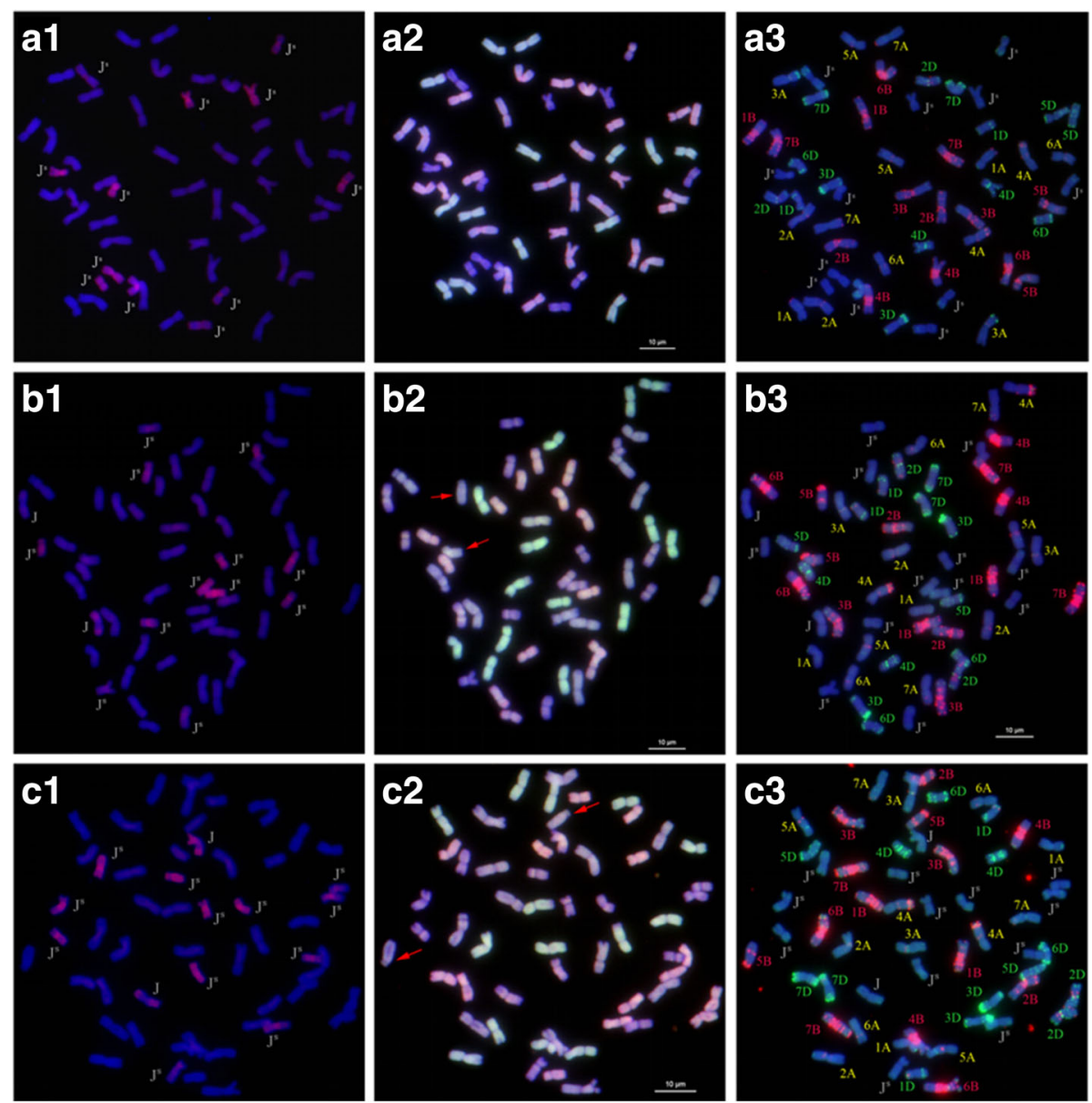

Fig. 1 GISH, McGISH and FISH patterns of SN0389, SN0398 and SN0406. GISH patterns of SN0389 (a1), SN0398 (b1) and SN0406 (c1): PS. spicata (St) genomic DNA labeled with Texas-Red-5-dCTP was used as the probe, and YN15 genome DNA was used to block. McGISH patterns of SN0389 (a2), SN0398 (b2) and SN0406 (c2): A-genomic DNA was labeled with green fluorescence, D-genomic DNA was labeled with red fluorescence and B-genomic DNA (gray) was used to block. As a result, Th. ponticum chromosomes showed purple signals. The FISH patterns of SN0389 (a3), SN0398 (b3) and SN0406 (c3): red signals were $(\mathrm{GAA})_{8}$ and green signals were pAs1 


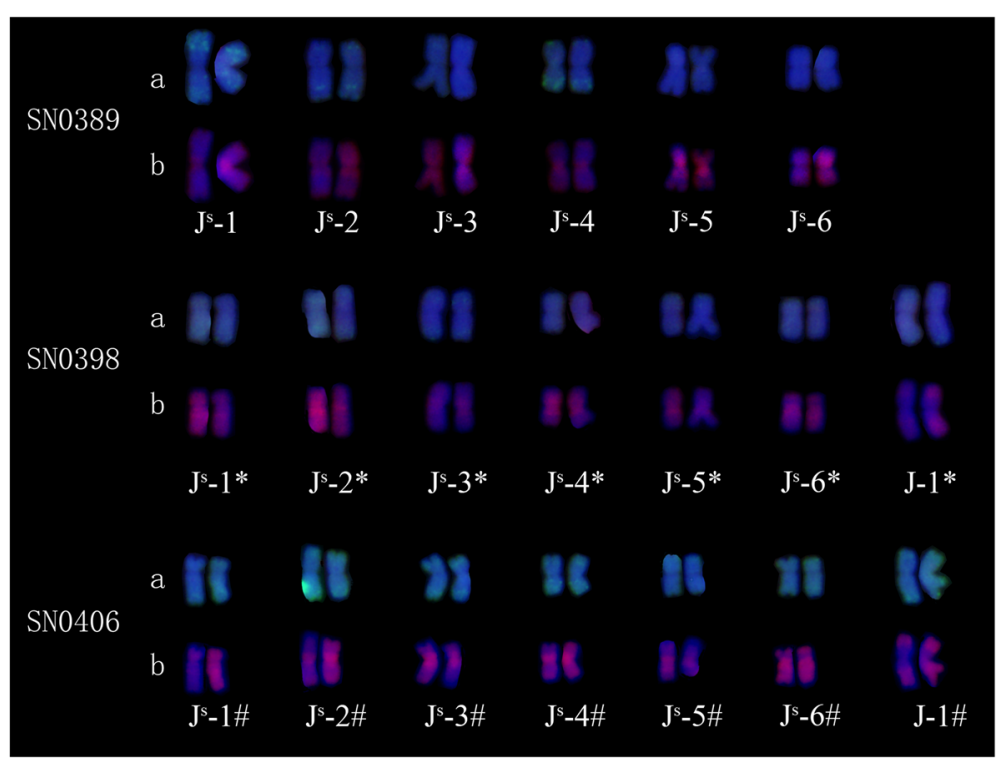

Fig. 2 FISH and GISH patterns of the alien chromosomes of SN0389, SN0398 and SN0406. a. FISH patterns of the alien chromosomes; b. GISH patterns of the alien chromosome. * behind the chromosome numbers indicate the $\mathrm{J}^{\mathrm{S}}$ and $\mathrm{J}$ chromosomes in SN0398. \# behind the chromosome numbers indicate the $\mathrm{J}^{S}$ and $\mathrm{J}$ chromosomes in SN0406. The alien chromosomes in SN0389, SN0398 and SN0406 with the same number did not mean the same chromosomes

chromosomes in SN0398 were similar with the $\mathrm{J}^{\mathrm{S}}-4^{\#}, \mathrm{~J}^{\mathrm{S}}-6^{\#}$ and $\mathrm{J}^{-} \mathrm{1}^{\#}$ chromosomes in SN0406 (Fig. 2). But as Th. ponticum contained 14 pairs of $\mathrm{J}^{\mathrm{S}}$ and 21 pairs of $\mathrm{J}$ chromosomes, and there were lack of specific in situ hybridization signals and specific molecular markers of each pairs of chromosomes in Th. ponticum, so it's difficult to identify the alien chromosomes in these three amphiploids.

Wheat chromosome variation of three partial amphiploids The wheat chromosome variation in the three partial amphiploids was analyzed using FISH and McGISH signals, and the results of the common wheat parent YN15 were used as a comparison (Fig. 3). For the A-genome chromosomes, the results showed that the additional red $(\mathrm{GAA})_{8}$ signals were detected at the terminal of 1AL in SN0389 (Fig. 3-E). Additional, apparently green, signals of the pAs1 probe were also found on 3AS of SN0389. Moreover, the green signal present in the terminal of 6AS in YN15 disappeared in the three partial amphiploids, and a pair of (GAA) $)_{8}$ signals of 6AL in SN0398 and SN0406 was absent (Fig. 3-E).

The red signals of $(\mathrm{GAA})_{8}$ in the B-genome chromosomes of SN0389, SN0398 and SN0406 were also changed a lot. For example, the $(\mathrm{GAA})_{8}$ signals at the long arm of 2B were absent in SN0398 and SN0406, while the red signal still remained in SN0389, and the absence was also observed on the satellite of $6 \mathrm{~B}$ in SN0389. Additional red signals were observed on 7BL of the three partial amphiploids. Furthermore, the $(\mathrm{GAA})_{8}$ signal on the terminal of 6BL in SN0389 was different from the other three materials and approached the end of 6BL in SN0389. Moreover, part of the 5B short arm in SN0398 and SN0406 was absent, compared with that of YN15 and SN0389. A few green signals of pAs1 were present on the 6BL chromosomes in $\mathrm{YN15}$, while they were not observed in the three germplasms (Fig. 3-E).

For the D-genome chromosomes in SN0389, SN0398 and SN0406, variations occurred in the $1 \mathrm{D}, 2 \mathrm{D}$ and $5 \mathrm{D}$ chromosomes. First, the red signals of $(\mathrm{GAA})_{8}$ on $1 \mathrm{DS}$ were absent in SN0389, while they were preserved in YN15, SN0398 and SN0406. Additionally, the green signals of pAs1, near the terminal of 2DL, were also absent in SN0398 and SN0406. Moreover, the pAs1 signal near the centromere of the 5D chromosomes in SN0389 and SN0398 was absent (Fig. 3-E).

\section{Phenotypic evaluation of three partial amphiploids}

The stripe rust resistance of SN0389, SN0398 and SN0406 at the seedling stage was evaluated in a greenhouse, while stripe and leaf rust resistance was evaluated under natural conditions (Table 1, Additional file 1: Figure S1). The results showed that SN0389, SN0398 and SN0406 showed good resistance to stripe rust race CYR32 in the seedling stage. At the adult-plant stage, these three partial amphiploids were immune to stripe rust and showed good resistance to leaf rust 

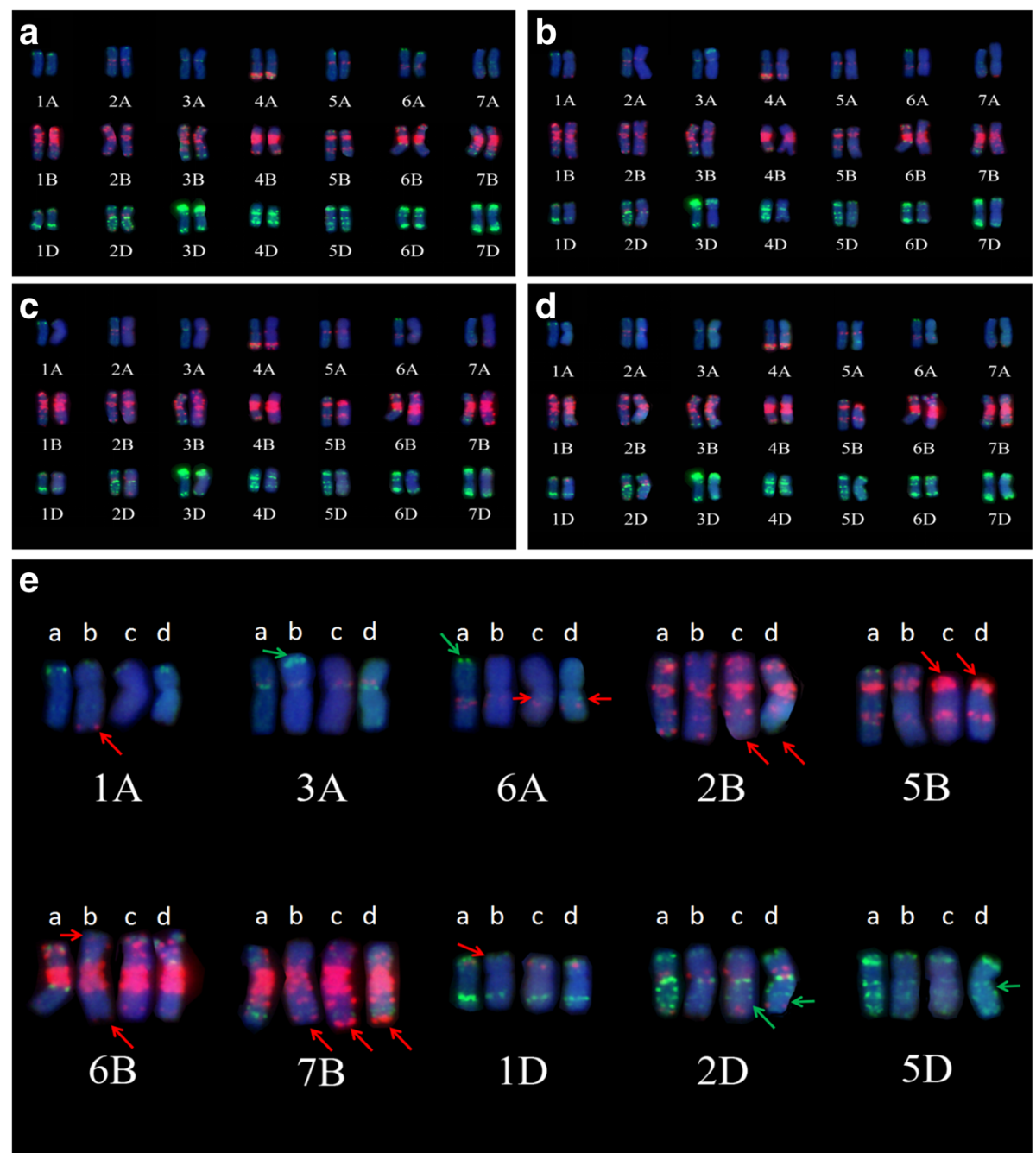

Fig. 3 FISH patterns of the wheat chromosomes in SN0389, SN0398 and SN0406 compared with the common wheat YN15. FISH patterns of YN15 (a), SN0389 (b), SN0398 (c), and SN0406 (d). In chromosomes H, I and J, on the left, were those in YN15, and those on the right were from the partial amphiploids. e, chromosome variations between YN15 and three partial amphiploids based on the FISH patterns. Among them, $a, b, c$ and $d$ indicated the corresponding chromosome of YN15, SN0389, SN0398 and SN0406 in turn

in the field. While its common wheat parent YN15 was susceptible to stripe rust and leaf rust and $T h$. ponticum was immune, we deduced that the resistance of partial amphiploids was derived from $T h$. ponticum.

Table 1 Stripe rust and leaf resistance evaluation of SN0389, SN0398 and SN0406

\begin{tabular}{llll}
\hline Materials & $\begin{array}{l}\text { Stripe rust CYR32 } \\
\text { Seedling stage }\end{array}$ & $\begin{array}{l}\text { Stripe rust } \\
\text { Adult-plant stage }\end{array}$ & $\begin{array}{l}\text { Leaf rust } \\
\text { Adult-plant stage }\end{array}$ \\
\hline Th. ponticum & 0 & 0 & 0 \\
YN15 & 4 & 4 & 4 \\
SN0389 & $0 ;$ & 0 & $0 ;$ \\
SN0398 & $0 ;$ & 0 & $0 ;$ \\
SN0406 & $0 ;$ & 0 & $0 ;$ \\
\hline
\end{tabular}

\section{Discussion}

Although Th. ponticum is closely related to wheat, it is difficult to obtain excellent germplasm materials directly by the hybridization between Th. ponticum and common wheat. Thus, wheat-Th. ponticum partial amphiploids, which contain the complete genomes of wheat but an incomplete genome (a set of chromosomes) of Th. ponticum, are used as crucial intermediate materials in the transfer of desirable genes from Th. ponticum to common wheat [3]. Several wheat-Th. ponticum amphiploids have been obtained, analyzed and exploited as alien sources of disease resistance in wheat improvement $[3,8,12,24,31]$.

In this study, we identified three novel wheat-Th. ponticum partial amphiploids with good rust resistance indpendent of stage. These three octoploid Trititrigia were developed in the common wheat cultivar YN15 
background, and they had good phenotypic characteristics, such as long spikes, advanced fluorescence, and higher cross-compatibility with wheat. Therefore, they could be used as bridge parents to cross with wheat to develop addition, substitution or translocation lines in order to provide new rust resistance germplasms for wheat breeding.

At both stages, YN15 was highly susceptible to stripe rust and leaf rust, whereas the three octoploid Trititrigia were immune at both stages. This indicated that SN0389, SN0398 and SN0406 possessed a resistant gene to rust that was derived from Th. ponticum. An analysis of the mitotic chromosomes showed that SN0389 had 42 wheat chromosomes and $12 \mathrm{Th}$. ponticum chromosomes, while SN0398 and SN0406 had 42 wheat chromosomes and 14 Th. ponticum chromosomes. The results indicated that the alien chromosome of SN0389 was $12 J^{\mathrm{S}}$. The alien chromosomes in SN0389 and SN0406 were not from a single genome of $\mathrm{J}^{\mathrm{S}}$ or $\mathrm{J}$, and the alien chromosome constitution of SN0398 and SN0406 was $12 J^{\mathrm{S}}+2 \mathrm{~J}$. Since there were 12 or 14 alien chromosomes in these three octoploid Trititrigia, it was difficult to deduce which chromosome the rust resistance gene was located on. We had hybridized the amphiploid with YN15, thus try to screen addition lines with different alien chromosomes in the derivative generations. Then it will be easier to identify which alien chromosome carrying the rust resistance gene. As there were lack of specific FISH or GISH signals and specific molecular markers of each chromosomes in Th. ponticum now, more work is needed to make that conclusion.

It is generally believed that only euploid amphiploids are genetically stable, while aneuploids often result in the loss of the added alien chromosomes [20]. Nevertheless, our results here show that the chromosome number of SN0389 was $2 \mathrm{n}=54$, and it only contained 12 alien chromosomes. However, the meiotic studies showed that SN0389 had a regular meiotic behavior after it was selfpollinated for several generations, and its chromosome number was still $2 \mathrm{n}=54$. Similar phenomena are also observed in partial amphiploid lines obtained from wheat $x$ Th. ponticum and wheat $\times$ Th. intermedium hybridizations $[8,10,24]$. For example, the partial amphiploid BE-1 contains 16 chromosomes derived from Th. ponticum and 40 wheat chromosomes, and the substituted wheat chromosome pair, as identified by FISH, was 7D [24]. Lines Zhong $1(2 n=52)$ and Zhong $2(2 n=54)$ both contain the complete wheat A, B and D genomes but with 10 and $12 \mathrm{Th}$. intermedium chromosomes, respectively [10]. Further research on the composition of these alien chromosomes and its compensation effect will be helpful in understanding the genetic relationship between the genome of Th. ponticum and wheat as well as be a benefit for transferring valuable traits from $T$. ponticum into wheat.
Using the in situ hybridization pattern of FISH and McGISH, chromosome variations in wheat were also detected. In this study, the structural variations also occurred in chromosomes 1A, 3A, 6A, 2B, 5B, 6B, 7B, 1D and 5D of SN0389, SN0398 and SN0406. The results indicated that during the formation of the partial amphiploids, various intergenomic rearrangements occurred. Some of the chromosome recombinations were caused by introgressed chromosome segments from Th. ponticum into common wheat chromosomes, while the introgressed segments were too small to detect by GISH. The other reason for the structural variations in the wheat chromosomes might also be that they were generated by recombination between different wheat chromosomes, such as homeologous chromosome recombination between the A-, B-, and D- genome genomic chromosomes that was interfered by the existence of the $T h$. ponticum chromosomes.

\section{Conclusions}

Three partial amphiploids with good resistance and different phenotypic traits were obtained in this study. The chromosome composition of the wheat-Th ponticum partial amphiploid was studied by means of GISH, McGISH and FISH. As a good source for improving disease resistance, these amphiploids could be used as promising crossing partners in wheat breeding programs, and resistant progenies of this partial amphiploid could be used as rust resistance sources in wheat improvement.

\section{Additional file}

Additional file 1: Figure S1. Reaction to stripe rust CYR32 of SN0389, SN0398 and SN0406. (TIF $1679 \mathrm{~kb}$ )

\section{Abbreviations}

FISH: Fluorescence in situ hybridization; GISH: In situ hybridization; mcGISH: Multicolor GISH; MI: Metaphase I

\section{Funding}

This work was supported by the National Key Research and Development Program of China (2016YFD0102000), National Natural Science Foundation of China (31671675) and Shandong Provincial Natural Science Foundation (ZR2015CM034)

Authors' contributions

YP performed experiments and prepared the manuscript. YC and YZ performed partial experiments and assisted with the manuscript writing. HW and YB performed partial experiments. XLdesigned the experiment and prepared the manuscript. All the authors reviewed and approved the manuscript.

Ethics approval and consent to participate Not applicable.

Competing interests

The authors declare that they have no competing interests. 


\section{Publisher's Note}

Springer Nature remains neutral with regard to jurisdictional claims in published maps and institutional affiliations.

Received: 1 March 2018 Accepted: 20 April 2018

Published online: 02 May 2018

\section{References}

1. Bao Y, Li X, Liu S, Cui F, Wang H. Molecular Cytogenetic Characterization of a new Wheat-Thinopyrum intermedium partial amphiploid resistant to powdery mildew and stripe rust. Cytogenet Genome Res. 2009;126(4):390-5.

2. Bao Y, Wu X, Zhang C, Li X, He F, Qi X, Wang H. Chromosomal constitutions and reactions to powdery mildew and stripe rust of four novel wheatThinopyrum intermedium partial amphiploids. J Genet Genomics. 2014; 41(12):663-6.

3. Chen Q, Conner R, Laroche A, Thomas J. Genome analysis of Thinopyrum intermedium and Thinopyrum ponticum using genomic in situ hybridization. Genome. 1998:41:580-6.

4. Chen S, Xia G, Quan T, Xiang F, Jin Y, Chen H. Introgression of salt-tolerance from somatic hybrids between common wheat and Thinopyrum ponticum. Plant Sci. 2004;167(4):773-9.

5. Dechyeva D, Schmidt T. Fluorescent in situ hybridization on extended chromatin fibers for high-resolution analysis of plant chromosomes. Plant Cytogenetics. 2016;1429:23-33.

6. Draz I, Abou-Elseoud M, Kamara A, Alaa-Eldein O, El-Bebany A. Screening of wheat genotypes for leaf rust resistance along with grain yield. Ann Agric Sci. 2015;60(1):29-39.

7. Dundas I, Zhang P, Verlin D, Graner A, Shepherd K. Chromosome engineering and physical mapping of the Thinopyrum ponticum translocation in wheat carrying the rust resistance gene Sr26. Crop Sci. 2015;55:648-57.

8. Fedak G, Chen Q, Conner RL, Laroche A, Petroski R, Armstrong KW. Characterisation of wheat-Thinopyrum partial amphiploids by meiotic analysis and genomic in situ hybridization. Genome. 2000;43:712-9.

9. Fu S, Lv Z, Qi B, Guo X, Li J, Liu B, Han F. Molecular cytogenetic characterization of wheat-Thinopyrum elongatum addition, substitution and translocation lines with a novel source of resistance to wheat Fusarium head blight. J Genet Genomics. 2012;39(2):103-10.

10. Han F, Liu B, Fedak G, Liu Z. Genomic constitution and variation in five partial amphiploids of wheat-Thinopyrum intermedium as revealed by GISH, multicolor GISH and seed storage protein analysis. Theor Appl Genet. 2004; 109(5):1070-6.

11. He F, Wang Y, Bao Y, Ma Y, Wang X, Li X, Wang H. Chromosomal constitutions of five wheat-Elytrigia elongata partial amphiploids as revealed by GISH, multicolor GISH and FISH. Comp Cytogenet. 2017;11(3):525-40.

12. Hu L, Li G, Zeng Z, Chang Z, Liu C, Yang Z. Molecular characterization of a wheat-Thinopyrum ponticum partial amphiploids and its derived substitution line for resistance to stripe rust. J Appl Genet. 2011;52(3):279-85.

13. Hu L, Li G, Zeng Z, Chang Z, Liu C, Zhou J, Yang Z. Molecular cytogenetic identification of a new wheat-Thinopyrum substitution line with stripe rust resistance. Euphytica. 2010;177(2):169-77.

14. Jauhar P, Peterson T, Xu S. Cytogenetic and molecular characterization of a durum alien disomic addition line with enhanced tolerance to Fusarium head blight. Genome. 2009;52(5):467-83.

15. Komuro S, Endo R, Shikata K, Kato A. Genomic and chromosomal distribution patterns of various repeated DNA sequences in wheat revealed by a fluorescence in situ hybridization procedure. Genome. 2013;56(3):131-7.

16. Kuzmanović L, Ruggeri R, Virili M, Rossini F, Ceoloni C. Effects of Thinopyrum ponticum chromosome segments transferred into durum wheat on yield components and related morpho-physiological traits in mediterranean rainfed conditions. Field Crop Res. 2016;186:86-98.

17. Li H, Wang X. Thinopyrum ponticum and Th. intermedium: the promising source of resistance to fungal and viral diseases of wheat. J Genet Genomics. 2009;36(9):557-65

18. Li X, Jiang X, Chen X, Song J, Ren C, Xiao Y, Gao X, Ru Z. Molecular cytogenetic identification of a novel wheat-Agropyron elongatum chromosome translocation line with powdery mildew resistance. PLoS One. 2017:12(9):e0184462

19. Liu L, Luo Q, Teng W, Li B, Li H, Li Y, Li Z, Zheng Q. Development of Thinopyrum ponticum-specific molecular markers and FISH probes based on SLAF-seq technology. Planta. 2018;247(5):1099-108.
20. Matzke MA, Scheid OM, Matzke AJM. Rapid structural and epigenetic changes in polyploid and aneuploid genomes. Bioessays. 1999;21:761-7.

21. Monneveux P, Reynolds M, Aguilar J, Singh R. Effects of the 7DL.7Ag translocation from Lophopyrum elongatum on wheat yield and related morphophysiological traits under different environments. Plant Breed. 2003; 122:379-84.

22. Muramatsu M. Cytogenetics of decaploid Agropyron elongatum (Elytrigia elongata) $(2 n=70) .1$. frequency of decavalent formation. Genome. 1990;33:811-7.

23. Niu Z, Klindworth D, Yu G, Friesen T, Chao S, Jin Y, Cai X, Ohm J, Rasmussen J, Xu SS. Development and characterization of wheat lines carrying stem rust resistance gene Sr43 derived from Thinopyrum ponticum. Theor Appl Genet. 2014;127(4):969-80.

24. Sepsi A, Molnar I, Szalay D, Molnar-Lang M. Characterization of a leaf rustresistant wheat-Thinopyrum ponticum partial amphiploids BE-1, using sequential multicolor GISH and FISH. Theor Appl Genet. 2008;116(6):825-34.

25. Sharma-Poudyal D, Chen X. Models for predicting potential yield loss of wheat caused by stripe rust in the U.S. pacific northwest. Phytopathology. 2011;101(5):544-54.

26. Shen $X$, Kong $L$, Ohm H. Fusarium head blight resistance in hexaploid wheat (Triticum aestivum)-Lophopyrum genetic lines and tagging of the alien chromatin by PCR markers. Theor Appl Genet. 2004;108(5):808-13.

27. Shen $\mathrm{X}, \mathrm{Ohm} \mathrm{H}$. Molecular mapping of Thinopyrum-derived fusarium head blight resistance in common wheat. Mol Breed. 2007;20(2):131-40.

28. Wang M, Peng Z, Li C, Li F, Liu C, Xia G. Proteomic analysis on a high salt tolerance introgression strain of Triticum aestivum/Thinopyrum ponticum. Proteomics. 2008:8(7):1470-89.

29. Wang RRC, Zhang XY. Characterization of the translocated chromosome using fluorescence in situ hybridization and random amplified polymorphic DNA on two Triticum aestivum-Thinopyrum intermedium translocation lines resistant to wheat streak mosaic or barley yellow dwarf virus. Chromosome Res. 1996:4:583-7.

30. Yang Z, Ren Z. Chromosomal distribution and genetic expression of Lophopyrum elongatum (Host) A. Löve genes for adult plant resistance to stripe rust in wheat background. Genet Resour Crop Evol. 2001;48:183-7.

31. Zheng Q, Lv Z, Niu Z, Li B, Li H, Xu S, Han F, Li Z. Molecular cytogenetic characterization and stem rust resistance of five wheat-Thinopyrum ponticum partial amphiploids. J Genet Genomics. 2014:41(11):591-9.

\section{Ready to submit your research? Choose BMC and benefit from:}

- fast, convenient online submission

- thorough peer review by experienced researchers in your field

- rapid publication on acceptance

- support for research data, including large and complex data types

- gold Open Access which fosters wider collaboration and increased citations

- maximum visibility for your research: over $100 \mathrm{M}$ website views per year

At BMC, research is always in progress.

Learn more biomedcentral.com/submissions 